\title{
Contribution de l'essai d'indentation à la caractérisation d'aciers nitrurés
}

\author{
Olivier Bartier, Rochdi El Abdia ${ }^{\mathrm{a}}$, Gérard Mauvoisin et Ali Nayebi \\ Laboratoire de Recherche en Mécanique Appliquée de l’Université de Rennes, IUT de Rennes, 3 rue du Clos Courtel, BP 90422, \\ 35704 Rennes Cedex 7, France
}

Reçu le 3 septembre 2002, accepté le 2 juillet 2004

\begin{abstract}
Résumé - Une nouvelle méthode de détermination des propriétés mécaniques d'aciers homogènes et d'aciers nitrurés présentant un haut gradient de dureté est présentée. Cette méthode est basée sur la modélisation de la courbe d'indentation $(F-\delta)$ obtenue avec un indenteur sphérique en fonction des paramètres de la loi de comportement élastoplastique de l'acier testé. Après avoir donné une méthode de détermination de la dureté $(H V)$ à partir des propriétés mécaniques d'un matériau homogène, le modèle proposé est validé expérimentalement sur divers aciers non durcis. Pour les aciers nitrurés étudiés, une loi des mélanges basée sur le rapport des énergies plastiques dissipées dans la couche durcie et dans le substrat lors de l'indentation est établie. Une procédure d'optimisation numérique nous permet d'obtenir l'épaisseur et la contrainte d'écoulement de la couche mince supposée homogène. À partir de ces résultats et en utilisant la méthode de détermination de la dureté, on peut accéder au profil de dureté des aciers nitrurés. Les résultats déduits sont comparés avec ceux obtenus expérimentalement par des essais classiques de filiation de dureté, et confirment l'intérêt de la méthode proposée.
\end{abstract}

Mots clés : Traitements thermiques / bi-matériaux / méthode d'indentation / méthode des éléments finis / dureté

\begin{abstract}
Indentation test contribution to the nitrided steel characterisation. A new approach to determine mechanical properties of homogeneous and nitrided steels with a high hardness profile is presented. This method is based on the modelling of the indentation curve $(F-\delta)$ obtained by a spherical indenter according to the elastoplastic behaviour law parameters of the tested steel. Firstly, a hardness determination method $(H V)$ is given, using the mechanical properties of a homogeneous material. This method is experimentally validated on various homogeneous steels. For the studied nitrided steels, a mixture law is established. This law is based on the plastic energy ratios dissipated in the hardened layer and the substrate during the indentation. A numerical optimisation procedure leads to have the thickness and the yield stress of the homogeneous thin film. From these values and hardness determination method, we can obtain the nitrided steels hardness profile. The deduced results are compared with those experimentally obtained by the standard micro-indentation tests and confirm the interest of the proposed method.
\end{abstract}

Key words: Heat treatments / bi-materials / indentation method / finite element method / hardness

\section{Introduction}

Les traitements thermiques de surface sont appliqués à beaucoup d'éléments de machines particulièrement exposés à la corrosion, au frottement et à la fatigue. Différents traitements thermiques ou thermochimiques (cémentation, nitruration, carbonitruration, trempe superficielle, ... ) permettent d'augmenter les caractéristiques mécaniques des couches superficielles.

\footnotetext{
a Auteur correspondant : relabdi@univ-rennes1.fr
}

Pour contrôler ces traitements, la méthode la plus communément utilisée dans l'industrie est la technique de filiation de dureté par micro-indentation Vickers. Cette technique possède comme inconvénient d'être destructive et de coût élevé à cause de la lenteur de la procédure. On peut trouver aussi comme moyen de contrôle les techniques non destructives, comme par exemple la méthode des ultrasons basée sur une corrélation entre épaisseur traitée et rétrodiffusion du signal ou la technique des courants de Foucault basée sur une corrélation entre épaisseur traitée et propriétés électromagnétiques. 


\section{Nomenclature}

\begin{tabular}{|llllll|}
\hline$D$ & Diamètre de l'indenteur & $\mathrm{mm}$ & $n$ & Coefficient d'écrouissage & \\
$a$ & Rayon de contact & $\mathrm{mm}$ & $\varepsilon_{\mathrm{pl}}$ & Déformation plastique & $\%$ \\
$\delta$ & Déplacement de l'indenteur & $\mu \mathrm{m}$ & $\varepsilon_{\mathrm{el}}$ & Déformation élastique & $\%$ \\
$\delta_{\mathrm{r}}$ & Profondeur de pénétration résiduelle & $\mu \mathrm{m}$ & $\alpha$ & Coefficient d'influence & \\
$\delta_{\max }$ & Profondeur de pénétration maximale & $\mu \mathrm{m}$ & $P_{\mathrm{av}}$ & Pression de contact moyenne & $\mathrm{MPa}$ \\
$\sigma$ & Contrainte & $\mathrm{MPa}$ & $F$ & Force appliquée & $\mathrm{N}$ \\
$\sigma_{y}$ & Contrainte d'écoulement & $\mathrm{MPa}$ & $S_{\mathrm{max}}$ & Aire maximale de contact & $\mathrm{mm}^{2}$ \\
$\nu$ & Coefficient de Poisson & $\mathrm{GPa}$ & $E_{\mathrm{pl}}$ & Énergie plastique & $\mathrm{J}$ \\
$E$ & Module d'Young & $\rho, \theta, z$ & Coordonnées cylindriques & $\mathrm{m}, \mathrm{rad}, \mathrm{m}$ \\
\hline
\end{tabular}

Cependant ces méthodes indirectes sont difficiles à mettre en œuvre et ne peuvent fournir que la profondeur de dureté, et non le profil de dureté $[1,2]$.

Du fait de l'imperfection de ces méthodes, notre laboratoire a mis au point récemment une méthode de contrôle rapide des traitements superficiels basée sur la proportionnalité entre les efforts mesurés lors d'un perçage et la dureté du matériau testé [3]. À partir d'un modèle analytique prenant en compte la géométrie des arêtes de coupe du foret et l'hétérogénéité de la résistance au perçage, le profil de dureté est obtenu d'une manière quantitative à partir de l'effort en perçage. Le modèle proposé conduit à de bons résultats lorsque le test est réalisé sur un acier cémenté ou carbo-nitruré, présentant un gradient de dureté relativement faible sur des épaisseurs allant de quelques dixièmes de millimètre à plusieurs millimètres. Par contre, lorsque le matériau traité présente un haut gradient de dureté à une faible distance de la surface comme dans le cas des aciers nitrurés, la méthode de caractérisation par perçage s'avère imprécise car les dimensions des forets ne permettent pas d'obtenir des résultats exploitables pour des épaisseurs de la couche traitée inférieures à $0,1 \mathrm{~mm}$.

L'objectif de la présente étude consiste à déterminer les propriétés mécaniques des aciers ayant subi des traitements thermiques et possédant une fine couche durcie à haut gradient de dureté en exploitant la courbe forcedéplacement $(F-\delta)$ obtenue non plus au moyen du test de perçage mais à l'aide d'un essai d'indentation à force croissante.

Dans cet article, nous proposons tout d'abord une méthode de détermination de la dureté $H V$ à partir des propriétés mécaniques d'un matériau.

Nous déterminons ensuite à l'aide de la méthode des éléments finis, un modèle pour l'évolution de la courbe d'indentation $(F-\delta)$ en fonction des paramètres $\left(\sigma_{y}\right.$ et $\left.n\right)$ de la loi de comportement élastoplastique d'un acier. Le modèle proposé est validé expérimentalement sur divers aciers.

Enfin, nous établissons pour un matériau bi-couche une loi des mélanges basée sur le rapport des énergies plastiques dissipées dans la couche traitée et dans le substrat lors de l'indentation. Une procédure d'optimisation numérique nous permet d'obtenir l'épaisseur et la contrainte d'écoulement de la couche mince supposée homogène. Nous comparons, pour différents matériaux et traitements de surface, les résultats obtenus par notre méthode et ceux obtenus classiquement par filiation de dureté.

\section{Méthode de détermination de la dureté HV à partir des propriétés mécaniques}

Les premiers travaux sur l'indentation ont été effectués dès le XIX ${ }^{\mathrm{e}}$ siècle dans le but de déterminer les paramètres élastiques du caoutchouc. Les théories utilisées pour ces études furent la théorie de Hertz [4] dans le cas des indenteurs sphériques et les équations de Sneddon [5] pour les indenteurs côniques.

Pour un métal supposé plastique, Tabor [6] fut le premier à donner une relation entre la dureté (en $\mathrm{MPa}$ ) Vickers $H V$ (ou la dureté Brinell $H B$ ) et la contrainte d'écoulement $\sigma_{y}$ :

$$
H V \approx 3 \sigma_{y} \quad \text { et } \quad H B \approx 2,8 \sigma_{y}
$$

Cette première observation expérimentale fut consolidée par une analyse théorique de l'écoulement plastique basée sur la méthode des lignes de glissement pour des indenteurs sphériques par Ishlinsky [7], pour des indenteurs côniques par Lockett [8], ou pour des indenteurs Vickers pyramidaux par Haddow et al. [9].

Ces théories confirment le fait que la dureté $H$ est de l'ordre de 3 fois $\sigma_{y}$ pour des indenteurs émoussés ou pointus. Cependant l'analyse basée sur le caractère purement plastique du matériau devient insuffisante pour des matériaux durs pour lesquels l'élasticité ne peut être négligée.

La méthode de détermination de la dureté $H V$, à partir des paramètres mécaniques, proposée dans ce chapitre est basée sur les résultats des recherches numériques, expérimentales et théoriques effectuées ces dernières années sur l'indentation des matériaux élastoplastiques [10-13]. Elle est donc applicable dans le cas des aciers.

La figure 1 représente la courbe de chargement en fonction de la profondeur de pénétration de l'indenteur $(F-\delta)$. Durant le chargement, la courbe suit généralement une allure puissance de la forme $F=$ $W . \delta^{m}$ dans laquelle $W$ et $m$ dépendent des propriétés mécaniques du matériau et de la géométrie de l'indenteur. 


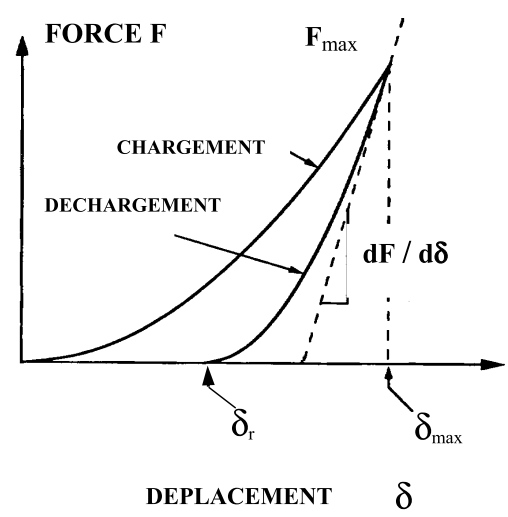

Fig. 1. Représentation schématique de la courbe forcedéplacement.

La courbe contrainte-déformation d'un métal peut être caractérisée par l'équation d'Hollomon du type :

$$
\sigma= \begin{cases}E \varepsilon_{\mathrm{el}} & \text { si } \sigma \leq \sigma_{y} \\ K \varepsilon^{n} & \text { si } \sigma \geq \sigma_{y}\end{cases}
$$

dans laquelle $\varepsilon$ représente la déformation totale $\left(\varepsilon=\varepsilon_{\mathrm{pl}}+\right.$ $\left.\varepsilon_{\text {el }}\right)$ et $n$ le coefficient d'écrouissage et $K=\sigma_{Y}^{1-n} \varepsilon^{n} E^{n}$. Le cas particulier $n=0$ indique un matériau parfaitement plastique.

La pression de contact moyenne $P_{\text {av }}$ est définie comme étant le rapport de la force appliquée $F$ par la projection de l'aire de contact $S$. Plusieurs simulations par éléments finis sur des matériaux élastoplastiques écrouissables (Giannakopoulos et al. [10]) et pour des aciers de coefficients d'écrouissage compris entre 0,1 et 0,4 (Jayaraman et al. [11]) montrent que cette pression moyenne peut être représentée par :

$\frac{P_{\mathrm{av}}}{E^{*}}=\frac{0,27}{E^{*}}\left(\sigma_{0,29}+\sigma_{y}\right) *\left(1+\ln \frac{E \cdot \tan \left(90^{\circ}-\frac{136^{\circ}}{2}\right)}{3 \sigma_{y}}\right)$

où :

- $136^{\circ}$ est l'angle d'acuité pour un indenteur Vickers,

- $E^{*}$ est le module effectif donné par la relation :

$$
E^{*}=\left[\frac{1-\nu^{2}}{E}+\frac{1-\nu_{\text {ind }}^{2}}{E_{\text {ind }}}\right]^{-1}
$$

$E, E_{\text {ind }}, \nu_{\text {ind }}$ et $\nu$ sont les modules d'Young et les coefficients de Poisson respectivement du matériau testé et de l'indenteur,

- $\sigma_{0,29}$ représente la contrainte pour un taux de déformation plastique de $29 \%$ pour un matériau soumis à une compression uniaxiale. Selon les auteurs [10-13], cette déformation plastique est représentative de la déformation plastique créée sous l'indenteur Vickers au même titre que la déformation équivalente donnée par Tabor dans le cas de l'indentation sphérique $\left(\varepsilon_{\mathrm{p}}^{\mathrm{av}}=0,4 a / D\right)$. Cette contrainte s'évalue grâce à l'équation (2) par :

$$
\sigma_{0,29}=K\left(0,29+\varepsilon_{\mathrm{el}}\right)^{n}
$$

Par ailleurs Giannakopoulos et al. [12] donnent l'expression de l'évolution de la charge en fonction du déplacement pour l'indentation Vickers qui permet d'écrire, dans le cas du chargement maximum, l'expression suivante :

$$
\frac{F_{\max }}{\delta_{\max }^{2}}=7,143\left(\sigma_{0,29}+\sigma_{y}\right)\left(-1+\ln \frac{E^{*}}{\sigma_{y}}\right)
$$

où :

- $F_{\text {max }}$ est la valeur maximale du chargement $F$,

- $\delta_{\max }$ est la pénétration maximale de l'indenteur.

Les auteurs ont établi cette relation à partir de simulations effectuées sur différents matériaux (aciers, fonte, alliages au nickel...) ayant un coefficient d'écrouissage inférieur à 0,15 [13].

En considérant les paramètres mécaniques du matériau indenté connus, on peut déduire à l'aide des équations (3) et (6) la valeur de la pénétration maximale $\delta_{\max }$ et de la pression de contact moyenne $P_{\mathrm{av}}$ obtenue lors de l'essai de dureté Vickers $H V_{30 \mathrm{~kg}}\left(F_{\max }=294 \mathrm{~N}\right)$.

En prenant en compte les effets de l'écrouissage lors de la formation du bourrelet et de l'affaissement de la matière au voisinage de l'indenteur, sur l'aire réelle de contact, Giannakopoulos et al. [12] proposent une relation entre l'aire maximale de contact $S_{\max }$ et la pénétration maximale $\delta_{\max }$ :

$$
\begin{aligned}
\frac{S_{\max }}{\delta_{\max }^{2}}= & 9,96-12,64\left(1-\frac{P_{\mathrm{av}}}{E^{*}}\right)+105,42\left(1-\frac{P_{\mathrm{av}}}{E^{*}}\right)^{2} \\
& -229,57\left(1-\frac{P_{\mathrm{av}}}{E^{*}}\right)^{3}+157,67\left(1-\frac{P_{\mathrm{av}}}{E^{*}}\right)^{4}
\end{aligned}
$$

La détermination de la valeur de la pénétration maximale $\delta_{\max }$ et de $P_{\text {av }}$ permet ainsi d'accéder à $S_{\max }$ grâce à l'équation (7).

D'autre part, le module effectif $E^{*}$ du couple indenteur-matériau est lié à $d F / d \delta$ par la relation suivante [14] :

$$
E^{*}=\frac{1}{c^{*} \sqrt{S_{\max }}}\left(\frac{d F}{d \delta}\right)
$$

où :

- $d F / d \delta$ est la pente de la courbe $F-\delta$ au début du déchargement (Fig. 1),

- $c^{*}$ est une constante dépendant du type d'indenteur. Elle est égale à 1,142 pour un indenteur pyramidal Vickers, 1,167 pour un indenteur de Berkovich et 1,128 pour un indenteur circulaire cônique.

La connaissance de $E^{*}, c^{*}$, et $S_{\max }$ rend alors possible l'obtention de la valeur $d F / d \delta$ à partir de l'équation (8). 
Tableau 1. $H V$ mesurée et $H V$ calculée à partir de $\sigma_{y}$ et $n$ obtenus par traction.

\begin{tabular}{ccccc}
\hline Aciers & $\begin{array}{c}\sigma_{y} \text { et } n \\
\text { obtenu par essai } \\
\text { de traction }\end{array}$ & $\begin{array}{c}n \text { obtenu par essai } \\
\text { de traction }\end{array}$ & $\begin{array}{c}\text { Dureté mesurée } \\
H V_{30 \mathrm{~kg}}\end{array}$ & $\begin{array}{c}\text { Dureté calculée } \\
H V_{30 \mathrm{~kg}}\end{array}$ \\
\hline 16NiCr6 & 345 & 0,17 & 206 & 202 \\
100C6 & 430 & 0,14 & 206 & 216 \\
35CrMo4 & 473 & 0,09 & 194 & 195 \\
35NiCr15 & 790 & 0,08 & 287 & 290 \\
X38CrMo V5 & 390 & 0,17 & 223 & 223 \\
X15CrNi 17-03 & 804 & 0,10 & 304 & 321 \\
X2CN18-10 & 200 & 0,21 & 155 & 155 \\
42 CrMo4 & 515 & 0,10 & 214 & 215 \\
C18 & 350 & 0,10 & 149 & 155 \\
C38 & 576 & 0,11 & 232 & 241 \\
C48 & 419 & 0,16 & 205 & 227 \\
C65 & 345 & 0,24 & 232 & 287 \\
C80 & 303 & 0,17 & 167 & 178 \\
\hline
\end{tabular}

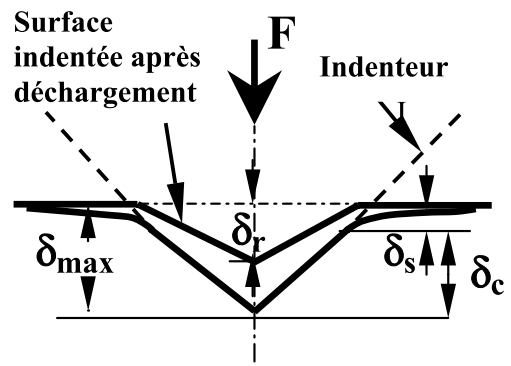

Fig. 2. Représentation schématique d'une section sous indentation.

À tout moment durant le chargement, le déplacement total $\delta$ de l'indenteur est donné par :

$$
\delta=\delta_{\mathrm{c}}+\delta_{\mathrm{s}}
$$

où $\delta_{\mathrm{c}}$ est la hauteur de la surface de contact entre l'indenteur et la matière et $\delta_{\mathrm{s}}$ est le déplacement de la surface au périmètre de contact (Fig. 2).

Après déchargement et retrait de l'indenteur, les déplacements élastiques étant restaurés, il ne subsiste alors que l'enfoncement rémanent représenté par $\delta_{\mathrm{r}}$.

Pour un indenteur cônique ou sphérique, cet enfoncement rémanent s'obtient à partir des paramètres calculés précédemment avec la relation de Sneddon suivante (cité dans [14]) :

$$
\delta_{\mathrm{r}}=\delta_{\max }-2 \frac{F_{\max }}{\left(\frac{d F}{d \delta}\right)}
$$

Le calcul de $\delta_{\mathrm{r}}$ permet ainsi de d'estimer la dureté Vickers $H V$ par l'équation (voir [15]) :

$$
H V=1,8544 \frac{F_{\max }}{49 \delta_{\mathrm{r}}^{2}}
$$

La figure 3 donne l'évolution théorique de la dureté en fonction de $n$ et $\sigma_{y}$ déterminée à l'aide de la méthode proposée. Comme on peut le remarquer sur cette figure, la dureté est globalement une fonction croissante de $\sigma_{y}$ et pour $n=0$ (matériau parfaitement plastique) c'est une

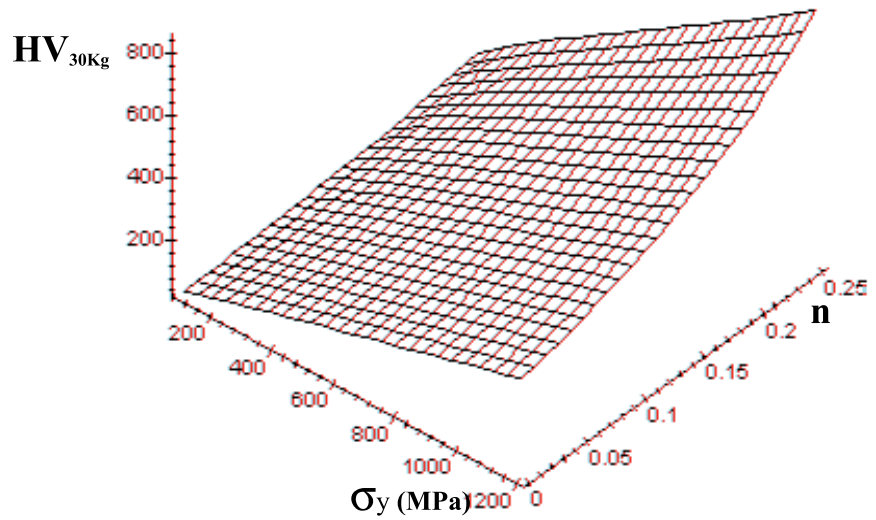

Fig. 3. Dureté $H$ en fonction de $\sigma_{y}$ et $n$.

fonction linéaire. C'est aussi une fonction croissante de $n$, dont l'évolution dépend de la valeur de $\sigma_{y}$.

Afin de tester la validité de la méthode proposée, nous avons entrepris une série d'essais sur plusieurs aciers. Le tableau 1 donne une comparaison entre les valeurs de dureté obtenues par un essai classique Vickers et celles calculées par la méthode présentée ci-dessus (Éqs. (3)-(11)) dans laquelle ont été introduites $n$ et $\sigma_{y}$ obtenues par un essai de traction. La figure 4 montre que les résultats obtenus par les deux méthodes sont très proches. La différence entre la dureté déterminée par la méthode proposée dans cet article et celle mesurée expérimentalement est inférieure à $10 \%$ pour tous les aciers testés sauf pour l'acier C65 (point le plus éloigné de la droite (Fig. 4)). Cet acier présente un coefficient d'écrouissage $(0,24)$ assez éloigné du domaine dans lequel la formule (11) a été établie $(n<0,15)$. Ceci pourrait indiquer que la méthode de détermination de la dureté proposée s'applique avec plus de succès lorsque l'exposant d'écrouissage ne dépasse pas trop 0,15 . Soulignons cependant, que la valeur de 0,15 est la limite du domaine étudié par les auteurs cités en référence, mais ne constitue pas forcément la limite du domaine de validité. D'ailleurs, plusieurs aciers étudiés paraissant dans le tableau 1, sont caractérisés correctement 


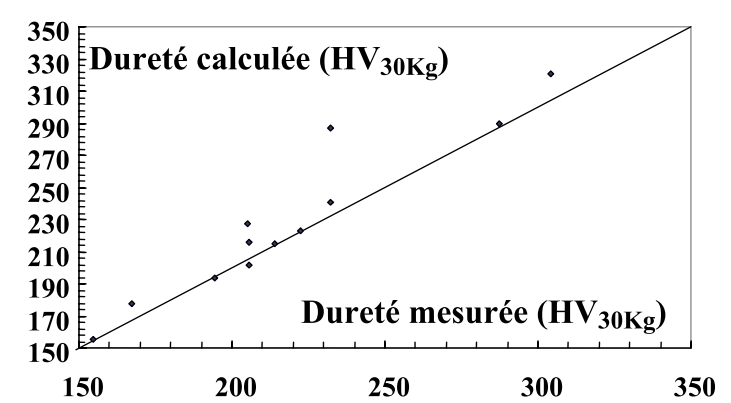

Fig. 4. Comparaison entre les duretés $H V_{30} \mathrm{~kg}$ calculées et mesurées.

malgré un exposant d'écrouissage supérieur à 0,15 (écart inférieur à $10 \%$ ).

Nous avons démontré dans ce paragraphe la validité de la méthode proposée pour déterminer la dureté $H V$ à partir des propriétés mécaniques $\left(E, \nu, n\right.$ et $\left.\sigma_{y}\right)$. Dans ce qui suit, nous présentons la procédure suivie pour obtenir la contrainte d'écoulement et le coefficient d'écrouissage d'un acier à l'aide de l'essai d'indentation à chargement croissant et non plus avec l'essai de traction, qui est la méthode la plus communément utilisée pour déterminer $\sigma_{y}$ et $n$.

\section{Méthode de détermination des paramètres mécaniques d'un matériau homogène}

On s'intéressera dans ce paragraphe au cas d'un matériau isotrope et homogène. Les résultats obtenus pour les matériaux homogènes seront utilisés ensuite lors du développement d'une méthode applicable aux matériaux nitrurés.

\subsection{Test de traction}

Les essais de traction sont réalisés sur différents matériaux dans le but d'obtenir des valeurs de références pour $n$ et $\sigma_{y}$. Les essais sont effectués sur une machine (type Lloyd LR30K), équipée d'un capteur d'effort de $30 \mathrm{KN}$ et d'un extensomètre HBM et pilotée en déplacement avec une vitesse d'avance de $2 \mathrm{~mm} . \mathrm{min}^{-1}$. La courbe de traction est obtenue à partir d'éprouvettes cylindriques normalisées de diamètre $6 \mathrm{~mm}$ et de longueur effective $24 \mathrm{~mm}$ (Fig. 5).

La figure 5 donne la courbe de traction pour l'acier 100C6. À partir de cette courbe, une procédure de minimisation des écarts entre la courbe expérimentale et la courbe théorique donnée par (2), permet d'obtenir les paramètres mécaniques $\sigma_{y}$ et $n$ de la loi d'Hollomon. Le tableau 1 regroupe les résultats de cette procédure sur les différents aciers testés. Une plage assez vaste de valeurs de $\sigma_{y}$ (de 200 à $800 \mathrm{MPa}$ ) et de $n$ (de 0,08 à 0,24$)$ a été étudiée.

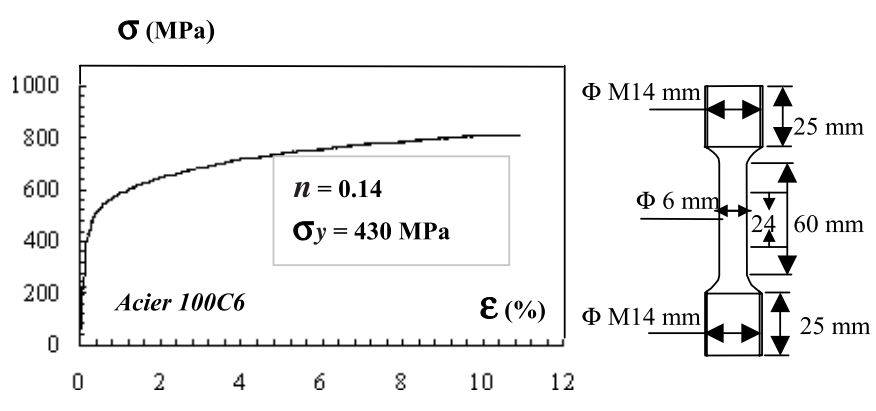

Fig. 5. Courbe de traction pour l'acier 100C6.

\subsection{Procédure d'indentation}

L'essai d'indentation à chargement croissant est effectué avec un indenteur sphérique de diamètre $D=$ 1,587 mm. La géométrie relativement simple de l'indenteur sphérique et la relative facilité avec laquelle il permet d'extraire les informations concernant les déformations plastique et élastique des matériaux testés expliquent le choix de ce type de pénétrateur.

Le test d'indentation est réalisé sur un macroduromètre instrumenté (Emcotest M4R-075) permettant de relever l'évolution de l'effort appliqué en fonction du déplacement de l'indenteur. Pour nos essais, la vitesse de déplacement de l'indenteur est de l'ordre de $8 \mathrm{~mm} \cdot \mathrm{min}^{-1}$ et le déplacement maximal de l'indenteur est de $100 \mu \mathrm{m}$.

Le macroduromètre utilisé possède une très grande rigidité. Sa conception est telle que seule les déformations en compression des éléments situés entre le pénétrateur et le capteur de déplacement sont à prendre en compte dans le calcul de cette rigidité. Une procédure de calibrage nous permet d'obtenir une courbe d'évolution $(F-\delta)$ très fiable. Les résolutions des capteurs de déplacement et d'effort sont respectivement de $1 \mu \mathrm{m}$ et de $1 \mathrm{~N}$. Afin d'améliorer la précision des résultats, la courbe d'évolution $(F-\delta)$ est obtenue à partir de la moyenne de trois tests d'indentation effectués en trois points différents de l'échantillon. Les échantillons utilisés lors de l'indentation et ceux destinés à la traction sont issus de la même barre d'acier.

\subsection{Modélisation numérique}

La simulation du test d'indentation par éléments finis sur Castem 2000 est réalisée en mode axisymétrique. Le comportement de l'indenteur est supposé élastique de grande rigidité $(E=600 \mathrm{GPa}, \nu=0,3)$ et celui de l'acier élastoplastique $(E=210 \mathrm{GPa}, \nu=0,3)$. La loi de comportement d'Hollomon et le critère d'écoulement de Von-Mises sont choisis pour l'acier testé. L'écrouissage du matériau est considéré isotrope. Afin que le modèle soit valide pour les principaux aciers utilisés dans l'industrie, les simulations sont effectuées pour des valeurs de $\sigma_{y}$ et de $n$ variant respectivement de 200 à $1600 \mathrm{MPa}$ et de 0 à 0,4 .

L'existence d'éléments de contact dans le code de calcul Castem 2000 permet de gérer automatiquement au 

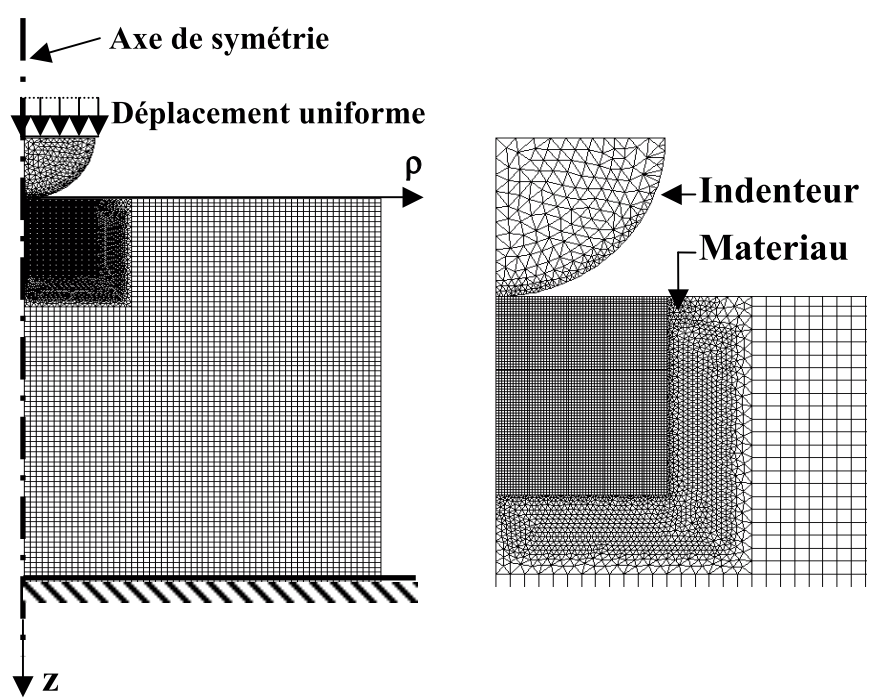

Fig. 6. Maillage éléments finis et zoom de la zone de contact.

cours du chargement la non pénétration et le décollement indenteur-matière. Plusieurs valeurs de coefficient de frottement comprises dans l'intervalle $[0 ; 0,5]$ ont été choisies afin d'étudier l'influence de ce paramètre sur l'évolution de la courbe $(F-\delta)$. Comme l'ont précédemment observé Taljat et al. [16], l'influence de ce coefficient sur la courbe $(F-\delta)$ a été constatée négligeable. Aussi pour la suite de l'étude, il est fixé à 0,2 pour l'ensemble des couples de valeurs de $\sigma_{y}$ et de $n$ étudiés.

Les conditions aux limites et de symétrie sont données dans la figure 6 .

Le maillage de l'indenteur et de l'éprouvette comportent 6316 éléments (éléments à 4 nœuds) et 5986 nœuds. Un total de 30 éléments d'interface est suffisant pour bien reproduire le contact entre l'indenteur et le matériau. Des simulations montrent qu'il n'y a pas de différence significative sur les résultats avec des maillages plus fins.

\subsection{Modèle proposé pour un matériau homogène}

Le but de ce paragraphe est de modéliser l'évolution de la courbe $(F-\delta)$ par une fonction dépendant des caractéristiques du matériau. Ceci afin de déterminer ensuite à l'aide d'une analyse inverse la contrainte d'écoulement $\sigma_{y}$ et le coefficient d'écrouissage $n$ d'un acier à partir d'un simple essai d'indentation.

L'ensemble des différentes simulations effectuées montre que la variation du déplacement de l'indenteur en fonction de l'effort appliqué suit une loi puissance que l'on peut modéliser par :

$$
F=A\left(\sigma_{y}, n\right) \times \delta^{B\left(\sigma_{y}, n\right)}
$$

où $A$ et $B$ sont deux fonctions dépendant de la contrainte d'écoulement $\sigma_{y}$ et du coefficient d'écrouissage $n$. L'approximation de l'ensemble des courbes $(F-\delta)$ obtenues

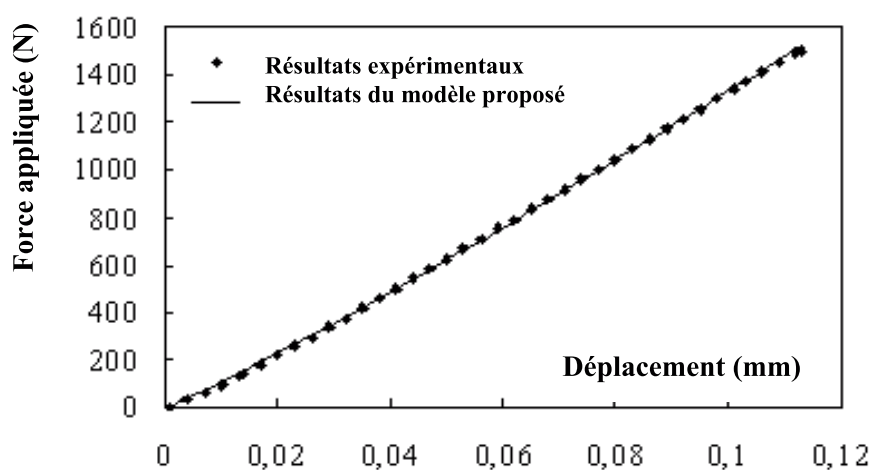

Fig. 7. Courbe force-déplacement pour l'acier 35CrMo4.

numériquement par la fonction (12) nous permet d'exprimer les fonctions $A$ et $B$ sous la forme suivante :

$$
\left.\begin{array}{l}
A=\left(-3294+22170 \times \sigma_{y}^{0,8}\right) \times e^{2,9 n \sigma_{y}} \\
B=\left(-0,151 \times \sigma_{y}+0,609\right) \times n+0,09 \times \sigma_{y}+0,975
\end{array}\right\}
$$

Notons que dans l'équation (13), la contrainte d'écoulement $\sigma_{y}$ est exprimée en GPa.

À partir des relations (12) et (13), un simple essai d'indentation et une procédure d'optimisation sont suffisants pour obtenir la contrainte d'écoulement $\sigma_{y}$ et le coefficient d'écrouissage $n$ de l'acier testé.

À titre d'exemple, la figure 7 représente la courbe $(F-$ $\delta$ ) obtenue pour l'acier $35 \mathrm{CrMo}$. Pour cet acier, on obtient $\sigma_{y}=457 \mathrm{MPa}$ et $n=0,10$ avec la méthode proposée (couplée avec l'essai d'indentation) et $\sigma_{y}=473 \mathrm{MPa}$ et $n=0,09$ avec l'essai de traction. Ce résultat et ceux donnés dans le tableau 2 montrent que notre méthode permet d'obtenir des caractéristiques mécaniques proches de celles obtenues par l'essai de traction. En conséquence, l'essai d'indentation permet d'obtenir la loi de comportement d'un acier en traction.

Les résultats obtenus pour les matériaux homogènes permettront de proposer dans ce qui suit, une méthode de détermination des caractéristiques mécaniques d'aciers nitrurés présentant un haut gradient de dureté.

\section{Caractérisation d'un matériau nitruré à haut gradient de dureté}

\subsection{Interaction entre la couche durcie et le substrat}

Après nitruration, l'épaisseur de la couche durcie obtenue peut varier de quelques centièmes de millimètres à quelques millimètres. Si l'interaction entre les éléments d'alliage de l'acier et l'azote est faible, la transition est progressive entre le maximum de dureté dans la couche de diffusion et la dureté du substrat. Par contre, s'il existe une interaction forte et une nucléation aisée des nitrures, la transition est brutale entre le maximum de dureté observé dans la couche de diffusion et la dureté du substrat, car la diffusion est gouvernée par la formation de nitrures [17]. Dans ce dernier cas, on parlera 
Tableau 2. $\sigma_{y}$ et $n$ obtenus par la méthode proposée et par traction.

\begin{tabular}{ccccc}
\hline$\sigma_{y}$ et $n$ & $\begin{array}{c}\sigma_{y} \text { obtenu par essai } \\
\text { de traction }\end{array}$ & $\begin{array}{c}\sigma_{y} \text { obtenu par indentation } \\
\text { et méthode proposée }\end{array}$ & $\begin{array}{c}n \text { obtenu par essai } \\
\text { de traction }\end{array}$ & $\begin{array}{c}n \text { obtenu par indentation } \\
\text { et méthode proposée }\end{array}$ \\
\hline 16NiCr6 & 345 & 423 & 0,17 & 0,15 \\
100C6 & 430 & 422 & 0,14 & 0,15 \\
35CrMo4 & 473 & 457 & 0,09 & 0,10 \\
35NiCr15 & 790 & 748 & 0,08 & 0,09 \\
X38CrMo V5 & 390 & 387 & 0,17 & 0,19 \\
X15CrNi 17-03 & 804 & 811 & 0,10 & 0,11 \\
X2CrNi18-10 & 200 & 276 & 0,21 & 0,18 \\
42CrMo4 & 515 & 479 & 0,10 & 0,13 \\
C18 & 350 & 325 & 0,10 & 0,12 \\
C38 & 576 & 492 & 0,11 & 0,13 \\
C48 & 419 & 429 & 0,16 & 0,15 \\
C65 & 345 & 384 & 0,24 & 0,22 \\
C80 & 303 & 308 & 0,17 & 0,17 \\
\hline
\end{tabular}

d'un bimatériau, constitué d'un film homogène à dureté constante et élevée, et d'un substrat à dureté moindre.

Lorsqu'on désire accéder aux caractéristiques mécaniques de la couche durcie à partir d'un essai d'indentation en surface, on se heurte généralement à l'interaction entre la couche durcie et le substrat. En effet, lorsque la charge est trop élevée pour l'épaisseur de la couche, la couche superficiellement durcie transmet la déformation au substrat moins dur. L'essai de microdureté ne conduit dans ce cas qu'à une mesure moyenne des caractéristiques du substrat et du film et non aux caractéristiques intrinsèques du film durci. Une solution possible pour caractériser seulement la couche est d'utiliser des chargements très faibles, afin que la déformation se limite uniquement à la couche durcie (méthode de nanoindentation) en respectant la règle du « $1 / 10^{\mathrm{e}} »[6]$, laquelle spécifie que le substrat ne perturbe pas les mesures de la dureté du film, si la profondeur d'indentation ne dépasse pas le $1 / 10^{\mathrm{e}}$ de l'épaisseur de la couche durcie. Mais cette règle n'est applicable qu'à la seule condition de connaître l'épaisseur de la couche.

Une autre alternative consiste à construire un modèle prenant en compte l'influence de la couche sur le substrat afin de dissocier les propriétés intrinsèques de ces deux couches. Nous choisissons cette deuxième alternative et proposons de prendre en compte l'interaction couchesubstrat par une loi des mélanges.

\subsection{Modèle proposé}

Afin de traduire l'influence du film et du substrat sur le comportement global du bimatériau lors de l'indentation, nous proposons la loi des mélanges suivante :

$$
\delta^{\mathrm{b}}=\alpha \delta^{\mathrm{f}}+(1-\alpha) \delta^{\mathrm{s}}
$$

dans laquelle les indices $b$, $f$ et $s$ sont associés respectivement au bimatériau, au film et au substrat. $\delta^{\mathrm{f}}$ représente le déplacement de l'indenteur si tout l'échantillon sous l'indenteur était constitué uniquement du «matériau film », $\delta^{\mathrm{s}}$ représente le déplacement de l'indenteur si tout l'échantillon était constitué uniquement du « matériau substrat » pour le même chargement et $\delta^{\mathrm{b}}$ est le déplacement mesuré au cours de l'indentation du bimatériau. $\alpha$ représente pour un effort donné, l'influence du film sur le déplacement mesuré lors de l'indentation du bimatériau.

En utilisant l'équation (12) pour les deux matériaux homogènes que sont le film et le substrat, nous pouvons écrire :

$$
\begin{aligned}
& \delta^{\mathrm{f}}=A\left(\sigma_{y}^{\mathrm{f}}, n^{\mathrm{f}}\right) * F^{B\left(\sigma_{y}^{\mathrm{f}}, n^{\mathrm{f}}\right)} \\
& \delta^{\mathrm{s}}=A\left(\sigma_{y}^{\mathrm{s}}, n^{\mathrm{s}}\right) * F^{B\left(\sigma_{y}^{\mathrm{s}}, n^{\mathrm{s}}\right)}
\end{aligned}
$$

Dans l'équation (14), $\alpha$ dépend de la contrainte d'écoulement, du coefficient d'écrouissage et de l'épaisseur du film $e^{\mathrm{f}}$ :

$$
\alpha=f\left(\sigma_{y}^{\mathrm{s}}, \sigma_{y}^{\mathrm{f}}, n^{\mathrm{s}}, n^{\mathrm{f}}, e^{\mathrm{f}}\right)
$$

$\alpha$ varie entre 0 et 1 . Lorsque le coefficient d'influence $\alpha$ est égal à 1 , seule l'influence du film est prise en compte. Lorsque $\alpha$ est nul seule l'influence du substrat est détectée (Éq. (14)). Plusieurs expressions de $\alpha$ ont déjà été proposées dans le passé [18]. Ces formulations ne donnant pas satisfaction dans le cas de nos matériaux, on propose d'adopter une approche énergétique pour définir $\alpha$ :

$$
\alpha=\frac{1}{2}\left(\frac{E_{\mathrm{pl}}^{\mathrm{ff}}}{E_{\mathrm{pl}}^{\mathrm{f}}}+\frac{E_{\mathrm{pl}}^{\mathrm{sf}}}{E_{\mathrm{pl}}^{\mathrm{s}}}\right)
$$

L'expression (18) est basée sur le résultat d'une superposition de deux systèmes hypothétiques représentés à la figure 8 dans laquelle :

- $E_{\mathrm{pl}}^{\mathrm{ff}}$ représente la partie de l'énergie plastique (créée au-dessous de l'indenteur) localisée dans le film si toute l'éprouvette était uniquement constituée du « matériau film »,

- $E_{\mathrm{pl}}^{\mathrm{sf}}$ représente la partie de l'énergie plastique localisée dans le film si toute l'éprouvette était uniquement constituée du « matériau substrat »,

- $E_{\mathrm{pl}}^{\mathrm{f}}$ représente l'énergie plastique totale contenue dans l'éprouvette si celle-ci était constituée uniquement du « matériau film », 

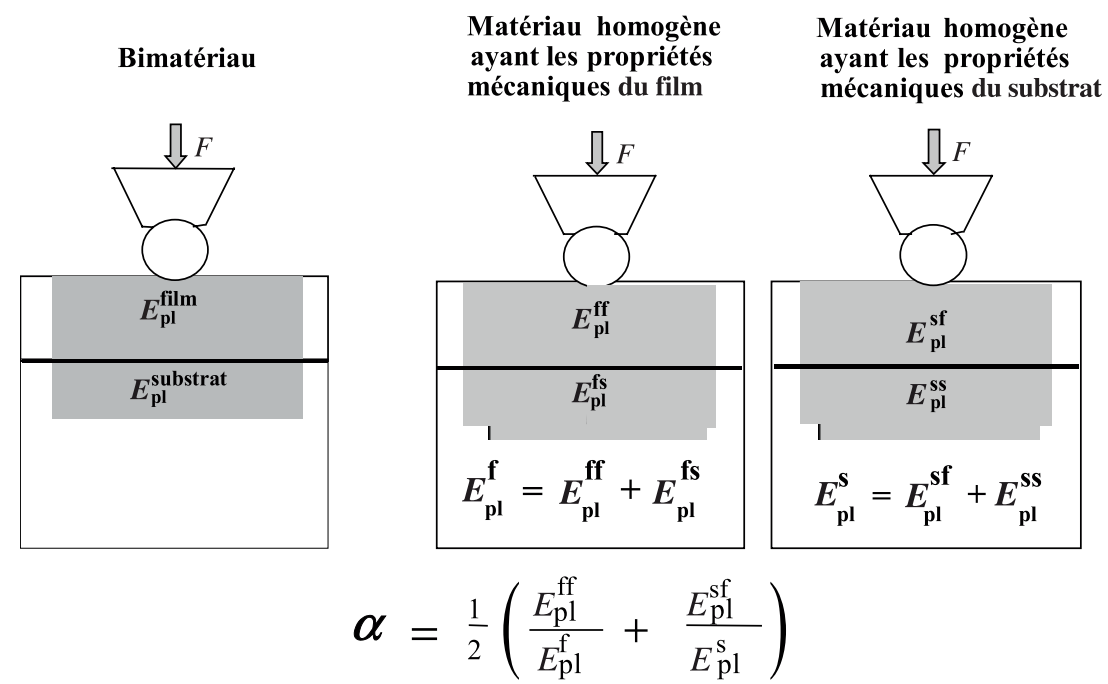

Fig. 8. Représentation schématique du modèle proposé.

- $E_{\mathrm{pl}}^{\mathrm{s}}$ représente l'énergie plastique totale contenue dans l'éprouvette si celle-ci était constituée uniquement du « matériau substrat ».

$\left(\mathrm{Si} E_{\mathrm{pl}}^{\mathrm{fs}}\right.$ représente la partie de l'énergie plastique localisée dans le substrat lorsque toute l'éprouvette est uniquement constituée du «matériau film» et si $E_{\mathrm{pl}}^{\mathrm{ss}}$ représente la partie de l'énergie plastique localisée dans le substrat lorsque toute l'éprouvette est uniquement constituée du matériau substrat, alors on a $E_{\mathrm{pl}}^{\mathrm{f}}=E_{\mathrm{pl}}^{\mathrm{fs}}+E_{\mathrm{pl}}^{\mathrm{ff}}$ et $\left.E_{\mathrm{pl}}^{\mathrm{s}}=E_{\mathrm{pl}}^{\mathrm{sf}}+E_{\mathrm{pl}}^{\mathrm{ss}}\right)($ Fig. 8).

Des simulations ont été effectuées en considérant des valeurs de $\sigma_{y}$ comprises entre 200 et $1600 \mathrm{MPa}$ et des valeurs de $n$ comprises entre 0 et 0,4 , ce qui représente une gamme d'aciers très large. À titre d'exemple, la figure 9, donnant l'évolution du paramètre $\alpha$ en fonction du chargement pour une simulation numérique effectuée avec $\sigma_{y}^{\mathrm{f}}=1200 \mathrm{MPa}, \sigma_{y}^{\mathrm{s}}=800 \mathrm{MPa}, n=n^{\mathrm{s}}=n^{\mathrm{f}}=0,2$ et un film d'épaisseur $0,4 \mathrm{~mm}$, montre que le modèle proposée (18), permet de prendre en compte correctement l'interaction couche-substrat. En effet, les valeurs de $\alpha$ obtenues avec notre modèle sont en bon accord avec celles exactes obtenues à partir de l'équation (14) :

$$
\alpha=\frac{\delta^{\mathrm{b}}-\delta^{\mathrm{s}}}{\delta^{\mathrm{f}}-\delta^{\mathrm{s}}}
$$

L'énergie plastique contenue dans un volume $V_{\mathrm{pl}}$ a pour expression :

$$
E_{\mathrm{pl}}=\int_{V_{\mathrm{pl}}} \int \sigma \mathrm{d} \varepsilon_{\mathrm{pl}} \mathrm{d} V_{\mathrm{pl}} \quad \text { avec } \quad \mathrm{d} V_{\mathrm{pl}}=\rho \mathrm{d} \rho \mathrm{d} \theta \mathrm{d} Z
$$

L'utilisation de cette équation, dont on trouvera la résolution numérique dans un précédent article [19], et des expressions (13), (14) et (18) montre que l'évolution du déplacement d'un indenteur de diamètre $D$ en fonction du chargement ne dépend, dans le cas d'un matériau bi-couche, que de la loi de comportement de la couche et du substrat et de l'épaisseur de la couche (l'épaisseur intervenant dans les bornes d'intégration).

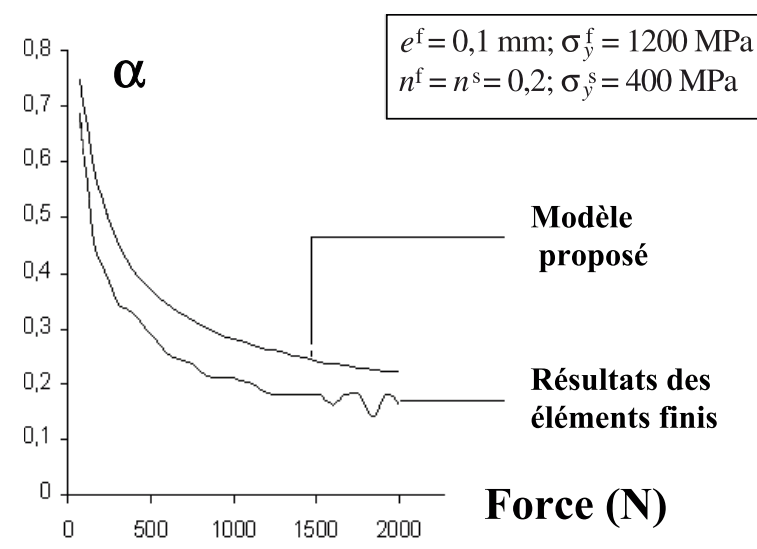

Fig. 9. Évolution de $\alpha$ en fonction du chargement.

Les paramètres mécaniques du substrat étant considérés connus, nous proposons de déterminer, par une procédure d'optimisation (minimisation de l'écart entre les courbes $(F-\delta)$ expérimentale et théorique (12)), la contrainte d'écoulement et l'épaisseur de la couche durcie.

\subsection{Identification des paramètres d'aciers traités}

L'expression (18) proposée pour déterminer l'interaction couche-substrat lors de l'essai d'indentation permet de considérer le bimatériau comme un assemblage de deux matériaux homogènes (film et substrat). Une indentation du matériau brut permet donc d'identifier, à l'aide des équations (12) et (13), les caractéristiques mécaniques du substrat du matériau nitruré.

D'autre part, il est montré que les traitements thermiques modifient $\sigma_{y}$ de façon substantielle, $n$ légèrement mais ne modifient pratiquement pas les autres caractéristiques mécaniques telles que le module d'Young [20]. En conséquence, si on considère d'une part que les caractéristiques mécaniques du matériau de base sont connues et d'autre part que les coefficients 


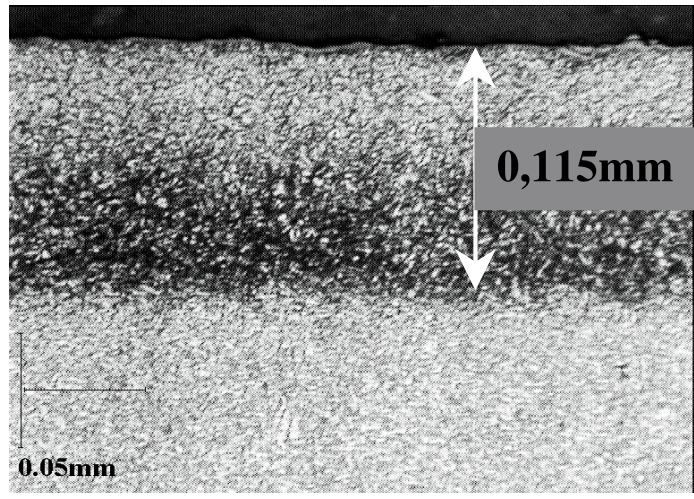

Fig. 10. Analyse métallurgique de l'acier nitruré $\mathrm{X} 38 \mathrm{CrMoV} 5$.

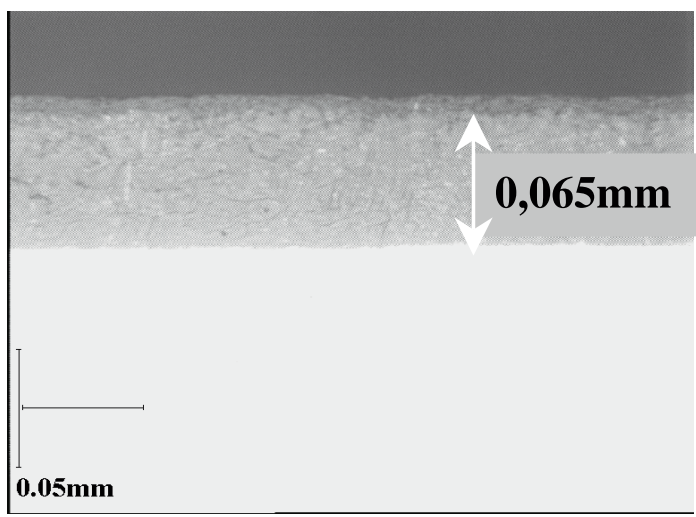

Fig. 11. Analyse métallurgique de l'acier nitruré X15CrNi1703 .

d'écrouissage du substrat et de la couche durcie sont les mêmes, seules $\sigma_{y}^{\mathrm{f}}$ et l'épaisseur de la couche $e^{\mathrm{f}}$ sont à déterminer dans l'équation (17).

La procédure à suivre pour contrôler la qualité d'un traitement de nitruration à partir de la courbe $(F-\delta)$ obtenue par indentation consiste d'abord à déterminer par une technique d'optimisation la contrainte d'écoulement et l'épaisseur de la couche durcie. Ensuite la connaissance de la contrainte d'écoulement $\sigma_{y}^{\mathrm{f}}$ permet d'obtenir la dureté de la couche nitrurée grâce aux équations (3)-(11).

À titre d'exemples, nous présentons ci-après les résultats obtenus sur deux aciers X38CrMoV5 et $\mathrm{X} 15 \mathrm{CrNi17}-03$ ayant tous deux subis un traitement de nitruration pendant $10 \mathrm{~h}$ à la température de $530{ }^{\circ} \mathrm{C}$. Les tests d'indentation sont effectués sur des échantillons cylindriques de $10 \mathrm{~mm}$ d'épaisseur. Seule une face de l'éprouvette a été soumise au traitement de nitruration. L'autre face servira pour déterminer les caractéristiques du substrat qui sont légèrement différentes de celles du matériau brut avant nitruration. Une analyse métallurgique montre (Figs. 10, 11) qu'on obtient bien un bimatériau constitué de deux couches distinctes respectivement d'épaisseur 0,115 mm pour l'acier X38CrMoV5 et d'épaisseur 0,065 pour l'acier X15CrNi17-03. L'essai expérimental d'indentation sur l'acier X38CrMoV5 effectué sur la face non nitrurée, conduit à une dureté

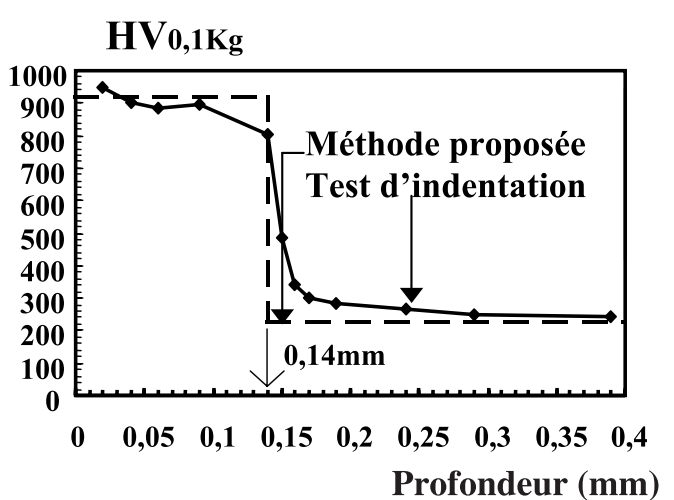

Fig. 12. Profil de dureté pour l'acier X38CrMoV5.

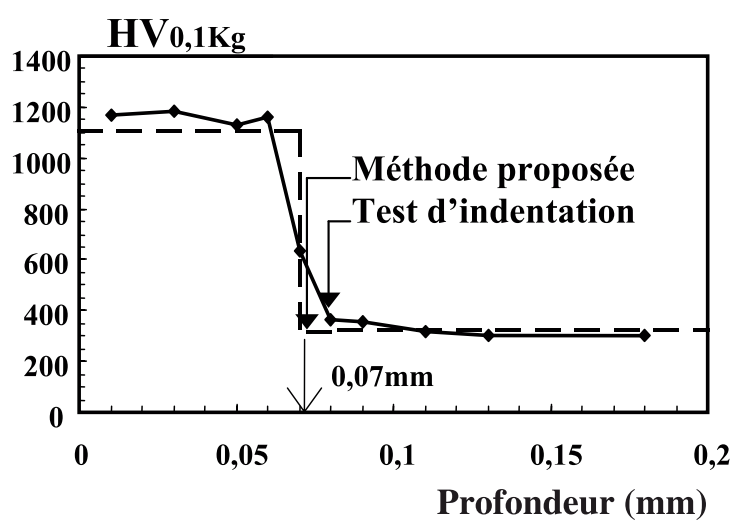

Fig. 13. Profil de dureté pour l'acier X15CrNi17-03.

du substrat de $H V_{0,1} \mathrm{~kg}=243$ (Fig. 12), et sur la face nitrurée à une valeur de $H V_{0,1 \mathrm{~kg}}=881$. Lorsqu'on applique la méthode proposée, celle-ci conduit respectivement aux valeurs de $H V_{0,1} \mathrm{~kg}=229$ pour le substrat et de $H V_{0,1} \mathrm{~kg}=921$ pour la couche durcie (Fig. 12). De plus, le modèle proposé donne une épaisseur de $0,14 \mathrm{~mm}$ pour la couche durcie, alors qu'une épaisseur de $0,115 \mathrm{~mm}$ est visualisée par analyse métallurgique (Fig. 10). Ce modèle permet aussi d'avoir la valeur du coefficient d'écrouissage égal à 0,145 .

La figure 12 indique que le modèle proposé conduit à une évolution similaire à celle donnée par la méthode classique de microindentation Vickers.

Les mêmes conclusions s'imposent pour l'acier X15CrNi17-03 (Fig. 13). En effet, l'essai expérimental d'indentation classique conduit aux valeurs de $H V_{0,1} \mathrm{~kg}=$ 305 pour le substrat et de $H V_{0,1} \mathrm{~kg}=1132$ pour la couche durcie et la méthode proposée donne $H V_{0,1 \mathrm{~kg}}=321$ pour le substrat et $H V_{0,1} \mathrm{~kg}=1106$ pour la couche durcie. On obtient une épaisseur de 0,07 mm (Fig. 13) (au lieu de 0,065 mm sur la coupe Fig. 11) et un coefficient d'écrouissage égal à 0,103 .

\section{Conclusion}

Dans cet article nous donnons tout d'abord une procédure permettant de retrouver la dureté $H V$ à partir de la connaissance de $\sigma_{y}$ et $n$ d'un acier. Les valeurs 
de dureté obtenues par cette démarche, en utilisant des valeurs de $\sigma_{y}$ et $n$ déterminées par traction, sont bien corrélées avec les valeurs de macrodureté mesurées sur de nombreux aciers.

Ensuite, une analyse basée sur des simulations de l'indentation de matériaux homogènes par éléments finis, a permis d'obtenir une relation qui relie le déplacement de l'indenteur et le chargement appliqué par le biais de deux fonctions $A$ et $B$ dépendant des caractéristiques mécaniques du matériau $\sigma_{y}$ et $n$ de la loi d'Hollomon. Ce modèle permet, par une procédure inverse, de déterminer ces caractéristiques mécaniques à partir d'une courbe d'indentation $(F-\delta)$. Les résultats obtenus sur plusieurs aciers sont proches de ceux obtenus par l'essai de traction. La procédure est rapide, elle ne nécessite pas d'éprouvette spécifique comme dans le cas du test de traction et elle s'avère non destructive.

Enfin, nous proposons une loi des mélanges dans laquelle le coefficient $\alpha$ représente l'influence de la couche durcie sur le déplacement mesuré au cours de l'indentation d'un matériau bicouche. On montre que ce coefficient peut être déterminé à partir des énergies de déformations plastiques dissipées dans la couche et dans le substrat. L'utilisation de cette loi des mélanges permet l'exploitation d'une courbe d'indentation relevée sur un bicouche et conduit à la détermination de $\sigma_{y}$, de l'épaisseur $e^{\mathrm{f}}$ de la couche durcie et finalement au profil de dureté. Les résultats sur des aciers nitrurés présentant un haut gradient de dureté sont comparés à ceux obtenus par une filiation de microdureté. Une observation au microscope de la section du matériau a également permis de confirmer la valeur de l'épaisseur de la couche durcie. Notons que contrairement à ces deux dernières techniques, la méthode proposée ne nécessite pas la découpe de l'échantillon.

Pour l'ensemble des aciers étudiés, la loi de comportement introduite dans le code d'éléments finis est la loi d'Hollomon $\left(\sigma=K \varepsilon^{n}\right)$. Si cette loi reproduit assez bien le comportement général des aciers, elle n'est pas dans tous les cas la plus adéquate. Notre prochaine étape sera d'affiner le modèle proposé en introduisant la loi qui traduit au mieux le comportement de chaque acier étudié.

Remerciements. Les auteurs tiennent à remercier la société NITRUVID (95100 Argenteuil) pour sa collaboration et son soutien matériel.

\section{Références}

[1] J. Rivenez, Contrôle non destructif du traitement par induction, Traitement Thermique 281 (1995) 39-41

[2] T. Lhermitte, Mesure non-destructive par courants de Foucault des profondeurs de carbonitruration sur des arbres secondaires de boîte de vitesses, Traitement Thermique 318bis (1999) 27-30
[3] G. Mauvoisin, F. Chagneau, De l'analyse de la résistance interne par perçage à la détermination du profil du dureté des aciers traités en surface, C. R. Ac. Sc. T.327, Série II, b (1999) Paris, 19-24

[4] H. Hertz, Uber die Berührung festischer körper, Z. Reine und Angewande Math. 92 (1882) 156-171

[5] I.N. Sneddon, The relation between load and penetration in the axisymmetric Boussineq problem for a punch of arbitrary profile, Int. J. Eng. Sci. 3 (1965) 47-57

[6] D. Tabor, The hardness of metal, Clarendon Press, Oxford, 1951

[7] A.J. Ishlinsky, Axisymmetric plastic flow in the Brinell test, Prik. Math. Mekh. 8 (1944) 201-224 (en russe)

[8] F.J. Lockett, Indentation of a rigid plastic material by a conical indenter, J. Mech. Phys. Solids 11 (1963) 345-355

[9] J.B. Haddow, W. Johnson, Indentation with pyramids, I : Theory, Int. J. Mech. Sci. 3 (1961) 229-238

[10] A.E. Giannakopoulos, P.L. Larson, R. Vestergaard, Analysis of Vickers indentation, Int. J. Sol. and Struct. 31(19) (1994) 2679-2708

[11] S. Jayaraman, G.T. Hahn., W.C. Oliver, C.A. Rubin, P.C. Bastias, Determination of monotonic stress-strain curve of hard materials from ultra-low-load indentation tests, Int. J. Structures 35(5 6.) (1998) 365-381

[12] A.E. Giannakopoulos, S. Suresh, Determination of elastoplastic properties by instrumented sharp indentation, Scripta Materiala 40(10) (1999) 1191-1198

[13] T.A. Venkatesh, K.J. Van Vliet, A.E. Giannakopoulos, S. Suresh, Determination of elastoplastic properties by instrumented sharp indentation: guidelines for property extraction, Scripta Materiala 42 (2000) 833-839

[14] W.C. Oliver, G.M. Pharr, An improved technique for determining hardness and elastic modulus using load and displacement sensing indentation experiments, J. Mater. Res. 7(6) (1992) 1564-1583

[15] J.R. Tuck, A.M. Korsunsky, S.L. Bull, R.I. Davidson, On the application of the work of indentation approach to depth-sensing indentation experiments in coated systems, Surf. Coat. Tech. 137 (2001) 217-224

[16] B. Taljat, T. Zacharia, F. Kosel, New analytical procedure to determine stress-strain curve from spherical indentation data, Int. J. Solids and Structures 35(33) (1998) 4411-4426

[17] C.V. Robino, O.T. Inal, Ion-nitriding behavior of severals low alloy steels, Materials Science and Engineering 78 (1983) 79-90

[18] D. Chicot, J. Lesage, Dureté des matériaux déposés en couches minces, Matériaux \& Techniques 10-11 (1994) $19-23$

[19] A. Nayebi, G. Mauvoisin, O. Bartier, R. El Abdi, Numerical and experimental analysis for determination of the mechanical properties of heat treated steels, Computational Steel Structure Technology, Civil-Comp Press (2000) 61-69

[20] A.E. Giannakopoulos, Total deformation, plane-strain contact analysis of macroscopically homogeneous compositionally graded materials with constant power-law strain hardening, J. Applied Mech. 64 (1997) 853-860 